months during the first year of treatment. Some of the patients received both drugs in different evolutionary periods of their disease. The study was approved by the Clinical Research Ethics Committee (CREC) of our hospital.

Results: Of the 42 patients included, 29 received $\mathrm{Pi}, 69 \%$ men and $31 \%$ women, with a mean age of 71 years (78\% ex-smokers). Baseline FVC was $2140 \mathrm{ml}$ (74.4\% of the predicted value) and DLCO was $40.8 \%$ with respect to the expected value. The absolute loss in FVC after 52 weeks of follow-up was $200 \mathrm{ml}$. $48.3 \%$ required treatment with glucocorticoids (GC) at some point, either due to exacerbations of the disease or as concomitant treatment. $65.5 \%$ presented some adverse reaction to $\mathrm{Pi}$, being gastrointestinal discomfort (GI) the most frequently observed, although mainly of self-limiting course, with the definitive suspension of the drug being necessary in 6 cases. As for the patients treated with $\mathrm{Ni}, 70.6 \%$ were men and $29.4 \%$ women, $82 \%$ ex-smokers, with an average age of 72 years. Baseline CVF value was $2480 \mathrm{ml}(83.8 \%$ of the predicted value) and DLCO value was $54.7 \%$. The decrease in FVC in absolute terms was $70 \mathrm{ml}$. Similarly, 4 patients required the use of GC at some point in the study. With regard to adverse reactions, $76.5 \%$ presented some type of adverse event, Gl discomfort being the most frequent, followed by increased transaminases and mild diarrhoea. The great majority were of limited duration, requiring the definitive suspension of the drug in 5 patients. Five patients treated with $\mathrm{Pi}$ died due to exacerbations of their disease.

Conclusions: This project supports, with data from usual clinical practice, the beneficial effect of the AF drugs available for the treatment of mild-moderate IPF. Both drugs have been shown to slow down the natural evolution of the disease, reducing the loss of FVC, a variable directly related to mortality. This therapy has acceptable safety margins. However, there are still no references regarding its administration in incipient and advanced stages of the disease nor on their combined use with each other or with immunomodulators for the control of immune mediated diseases.

Acknowledgements: To the nurses and all members of the Pneumology Service for their collaboration in the follow-up of the patients included in this study.

Disclosure of Interest: None declared

DOI: 10.1136/annrheumdis-2018-eular.7208

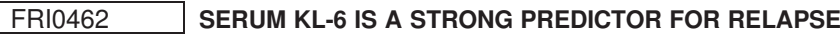 OF MYOSITIS-ASSOCIATED INTERSTITIAL LUNG DISEASE}

S. Takanashi, N. Nishina, M. Nakazawa, Y. Kaneko, T. Takeuchi. Rheumatology, Keio University School of Medicine, Shinjuku-ku, Tokyo, Japan

Background: Polymyositis (PM), dermatomyositis (DM) and clinically amyopathic DM (CADM) are autoimmune myositis which can be associated with interstitial lung disease (ILD). ${ }^{1,2}$ The relapse rate of ILD is high, reported as approximately $20 \%-55 \%$. $^{3,4}$ Since relapses result in decreased pulmonary function, it is important to identify the predictive factors for the relapse.

Objectives: The aim of this study was to elucidate the predictive factors for the relapse of ILD associated with myositis (PM/DM/CADM).

Methods: We conducted an observational retrospective study. Patients with myositis-associated ILD who have ever visited our institution between 2002-2017 and achieved remission once were enrolled. Patients who died before remission were excluded. We collected their clinical information from medical records. We compared patient characteristics between relapse group and non-relapse group by Fisher's exact test or Mann-Whitney $U$ test at first. Relapse was defined as exacerbation of radiological findings of which doctor-in-charge decided to intensify therapy for ILD. We performed Kaplan-Meier analysis to compare the relapse-free survival for the characteristics that had significant differences between two groups. To perform Kaplan-Meier analysis, continuous variables were converted to dichotomous variables for analysis by setting cut-off values determined by receiver-operating characteristics (ROC). Then, using each characteristic which showed significant difference in Kaplan-Meier analysis, we conducted Cox's proportional hazard analysis for multivariate analysis. For relapse group, we examined the changes of serum KL-6 levels from the initial treatment of myositisassociated ILD to the relapse of ILD. We calculated the average of serum KL-6 levels of 3 months and 6 months before relapse, respectively, then compared them with KL-6 levels at the time of relapse.

Results: Seventy-two patients with myositis-associated ILD at our institution were enrolled. Among 72 patients, 24 experienced relapse (relapse group) and 48 did not experience relapse (non-relapse group). Median observational period was 31.5 months and 39.0 months, respectively. Median levels of serum KL-6, the rate of patients who had upper lung field (ULF) lesion by CT, and anti-ARS antibody prevalence were significantly higher in relapse group than in non-relapse group (1870 vs $935 \mathrm{U} / \mathrm{mL}, \mathrm{p}=0.003$; 62 vs $27 \%, \mathrm{p}=0.01$; 88 vs $60 \%, p=0.03$, respectively). Median levels of\%VC was significantly lower in relapse group than in nonrelapse group ( 65.7 vs $81.2 \%, p=0.02$ ). By $R O C$ analysis, the cut-off levels of serum KL-6 and\%VC were determined as $1359 \mathrm{U} / \mathrm{mL}$ and $70.5 \%$, respectively. Kaplan-Meier analysis showed serum KL-6 >1359 U/mL $(p=0.02)$, anti-ARS antibody $(p<0.05), \%$ VC $<70.5(p=0.004)$, and ULF lesions $(p=0.01)$ were significantly related to the relapse (figure 1 ). Multivariate analysis revealed only serum $\mathrm{KL}-6>1359 \mathrm{U} / \mathrm{mL}$ was an independent risk factor for relapse (hazard ratio: 4.9 $(95 \% \mathrm{Cl} 1.0-24.0), \mathrm{p}<0.05)$ among the 4 characteristics. At the time of the relapse, serum KL-6 levels were increased $37 \%$ from the 3 months average and $51 \%$ from the 6 months average.


Conclusions: Serum KL-6 was a strong predictor for relapse of myositis-associated ILD.

REFERENCES:

[1] N Engl J Med. 1975;292:344.

[2] J Am Acad Dermatol. 2002;46:626.

[3] Autoimmunity 2006;39:233.

[4] Rheumatology (Oxford) 2015;54:39.

Disclosure of Interest: None declared DOI: 10.1136/annrheumdis-2018-eular.2045

\section{FRI0463 A NEW COMPUTED TOMOGRAPHY INDEX FOR QUANTIFICATION OF INTERSTITIAL LUNG DISEASE IN SYSTEMIC SCLEROSIS IS ASSOCIATED WITH LUNG FUNCTION PARAMETERS IN THE SHORT TERM FOLLOW-UP}

S. Vettori ${ }^{1}$, M. Bocchino ${ }^{2}$, D. Bruzzese ${ }^{3}$, M. D'Alto ${ }^{4}$, P. Argiento ${ }^{4}$, V. Giacco ${ }^{1}$, E. Romeo ${ }^{4}$, A. Capaccio ${ }^{2}$, G. Rea ${ }^{5}$, T. Valente $^{5}$, A. Sanduzzi-Zamparelli ${ }^{2}$, G. Valentini ${ }^{1}$. ${ }^{1}$ Biochemistry, Biophysics, and General Pathology, University of Campania Luigi Vanvitelli; ${ }^{2}$ Pneumology Unit, ${ }^{3}$ Biostatistics, University of Naples Federico II; ${ }^{4}$ Cardiology Unit, University of Campania Luigi Vanvitelli; ${ }^{5}$ Radiology Unit, Monaldi Hospital, Naples, Italy

Background: New computer-assisted methods for the objective quantification of interstitial lung disease (ILD) at computed tomography (CT), based on the evaluation of mean lung attenuation (MLA), skewness and kurtosis have been recently investigated in Systemic Sclerosis (SSc). We developed a computerised integrated index (CII) based on a weighted evaluation of MLA, skewness and kurtosis and investigated its reliability for the quantitative assessment of SSc-ILD and its associations with lung function parameters in a cross-sectional study.

Objectives: To identify the Cll cut off value with the highest sensitivity and specificity for CT-detected ILD and to investigate its impact on lung function parameters over-time of baseline assessed CII.

Methods: SSc patients meeting the new ACR/EULAR classification criteria, who had undergone a volumetric lung CT study from July 1 st 2014 to June 30th 2015 had been evaluated at baseline for ILD quantification by Goh et al. method and the previously referred dedicated software and had their CII calculated, were enrolled in a prospective study including complete clinical, serological, and functional assessment at baseline and at 1 year follow-up (FU).

Results: Thirty-nine out of $83(47 \%)$ SSc patients (79 females, 4 males; mean age $56.4 \pm 11.3$ years; median disease duration 12 years ${ }^{2-54} 18$ diffuse cutaneous and 65 limited cutaneous SSc) had evidence of ILD as assessed by volumetric CT of the lungs at baseline. CII in patients with ILD was significantly lower than in those without ILD $(-0.4929 \pm 0.9933$ versus $0.4145 \pm 0.8059 \mathrm{HU} ; \mathrm{p}<0.0001)$. ROC analysis revealed that the best discriminating CII value for ILD was 0.1966 
sensitivity 0.81 ( $95 \%$ C.I. 0.68 to 0.92$)$; specificity 0.66 ( $95 \%$ C.I. 0.52 to 0.80$)$. Out of the 44 ILD negative patients, $22(50 \%)$ presented a Cll value lower than the cutoff, and 13 of them (59\%) were found to have a diffusing lung capacity for CO (DLCO) $<80 \%$ of predicted. At 1 year FU, the Cll was significantly correlated with total lung capacity -TLC $(r=0.45, p=0.004)$ and DLCO $(r=0.29, p=0.045)$. Out of the 22 patients with a $\mathrm{Cll}<0.1966$ but no ILD at visual evaluation, $11(50 \%)$ developed a FVC decline at 1 year, and $8(36.7 \%)$ a DLCO decline.

Conclusions: Here we confirm that quantitative computer-assisted CT of the lungs could be a reliable method for SSc-ILD evaluation and found that it could also be useful in predicting the evolution of lung function in the short-term FU.

Disclosure of Interest: None declared

DOI: 10.1136/annrheumdis-2018-eular.7559

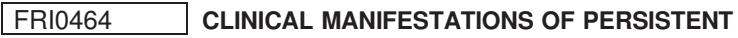 ANTIPHOSPHOLIPID ANTIBODIES IN SYSTEMIC SCLEROSIS PATIENTS}

S. Myriam ${ }^{1,2,3}$, V. Sobanski ${ }^{1,2,4}$, A. Lemaire ${ }^{1,2}$, J. Giovannelli $^{4}$, E. Hachulla ${ }^{1,2,4}$ B. Lopez ${ }^{4,5}$, M. Lambert ${ }^{1,2,4}$, P.-Y. Hatron ${ }^{1,2}$, S. Dubucquoi ${ }^{4,5}$, D. Launay ${ }^{1,2,4}$. ${ }^{1}$ Département de Médecine Interne et Immunologie Clinique; ${ }^{2}$ Centre national de référence maladies systémiques et auto-immunes rares (sclérodermie systémique), CHU Lille; ${ }^{3}$ Univ Lille; ${ }^{4}$ Lille Inflammation Research International Center (LIRIC), Univ. Lille,U995; ${ }^{5}$ Département d'Immunologie centre de biologie pathologie, CHU Lille, Lille, France

Background: Antiphospholipid antibodies (aPL) can be detected in systemic sclerosis (SSc) patients. While their clinical signification is well known in the primary antiphospholipid syndrome (APS), their frequency and clinical association remain debated matter of issue. In a previous study, we reported the prevalence and their clinical association of a single aPL testing a French cohort of SSc patients. Yet, the persistence of aPL during follow-up of patients has never been assessed.

Objectives: This study aimed to assess i. the persistence of aPL at least 12 weeks after the first dosage and ii. the clinical associations.

Methods: At least 12 weeks apart after the first test, 213 SSc patients were consecutively tested for lupus anticoagulant (LA), anticardiolipin (aCL) and antiB2glycoproteine 1 (anti-B2Gp1) antibodies. aCL and anti- $\beta 2 \mathrm{Gpl}$ were measured using commercial ELISA assays (Orgentec, Trappes, France), positivity was defined as $\geq 10 \mathrm{UGPL} / \mathrm{mL}(\mathrm{aCL})$ or $\geq 10 \mathrm{UA} / \mathrm{mL}$ (anti- $\beta 2 \mathrm{Gpl})$. Clinical associations were studied using binomial logistic regressions.

Results: The mean time between two tests was $13.2 \pm 6.3$ months. One or more type of aPL was persistent in 7 patients (3\%). Patients with persistent aPL developed more frequently venous thrombosis (VT) [4/7 (57\%) versus 23/205 (11\%), $\mathrm{p}=0.006]$. Arterial or/and venous thrombosis were more common in patients with persistent aPL than other patients [4/7 (57\%) versus 33/205 (16\%), p<0.001]. Persistent aPL was also associated with miscarriages [4/6 (67\%) versus $33 / 155$ $(21 \%), p=0.023]$.

Association between aPL positivity persistance and clinical manifestations

\begin{tabular}{|l|c|c|c|}
\cline { 2 - 4 } \multicolumn{1}{c|}{} & aPL positivity & aPL negativity & $\mathrm{p}$ \\
\hline Arterial or venous thrombosis & $4(57.1 \%)$ & $33(16.1 \%)$ & $<0.001$ \\
\hline Venous thrombosis & $4(57.1 \%)$ & $23(11.2 \%)$ & 0.006 \\
\hline Arterial thrombosis & $1(14.3 \%)$ & $14(6.8 \%)$ & 0.406 \\
\hline Miscarriage & $4(66.7 \%)$ & $32(20.6 \%)$ & 0.023 \\
\hline Digital ulceration & $0(0 \%)$ & $40(19.8 \%)$ & 0.351 \\
\hline PAH & $1(14.3 \%)$ & $13(6.7 \%)$ & 0.402 \\
\hline
\end{tabular}

Conclusions: The prevalence of persistent aPL in our cohort was $3 \%$. VT and miscarriages were associated with persistent $\mathrm{aPL}$.

\section{REFERENCES:}

[1] Parodi A, Drosera M, Barbieri L, Rebora A. Antiphospholipid antibody system in systemic sclerosis. Rheumatology (Oxford) 2001;40:111-12.

[2] Sanna G, Bertolaccini ML, Mamali A, et al. Antiphospholipid anti- bodies in patients with scleroderma: prevalence and clinical signifi- cance. Ann Rheum Dis 2005.

[3] Marie I, et al. Anticardiolipin, ab2GPI antibodies and lupus-like anticoagulant in SSc.

[4] Miryakis S, Lockshin MD, Atsumi T, et al. International consensus statement on preliminary classification criteria for definite antiphospholipid syndrome (APS). J Thromb Haemost 2006;4:295.

Disclosure of Interest: None declared

DOI: 10.1136/annrheumdis-2018-eular.6640

\section{FRI0465}

QUANTITATIVE ASSESSMENT OF INTERSTITIAL LUNG DISEASE IN IDIOPATHIC INFLAMMATORY MYOPATHIES: VISUAL SCORE AND COMPUTERISED PROCESSING ANALYSIS OF HIGH RESOLUTION COMPUTERISED TOMOGRAPHY

S. Barsotti ${ }^{1,2}$, C. Romei ${ }^{3}$, E. Cioffi ${ }^{2}$, E. Perrone ${ }^{3}$, C. Giovannetti ${ }^{2}$, M. Mosca $^{2}$,

F. Falaschi ${ }^{3}$, R. Neri' ${ }^{2}{ }^{1}$ Department of medical biotechnologies, University of Siena, Siena; ${ }^{2}$ Rheumatology Unit, University of Pisa; ${ }^{3}$ Radiology Unit, Pisa University Hospital, Pisa, Italy

Background: High resolution computed tomography (HRCT) is an essential technique for the characterisation of interstitial lung disease (ILD) in patients with idiopathic inflammatory myopathies (IIM). Although several visual scores are available for a semiquantitative evaluation of ILD, an automatic quantitative analysis of lung involvement may represent a valuable improvement to reproducibly determine the extent of the disease.

Objectives: To compare the semiquantitative visual score to a volumetric texture and local volumetric histogram feature-based analysis software (CALLIPER) to quantify the lung involvement in IIM patients.

Methods: 21 consecutive IIM patients (Bohan and Peter criteria) who underwent a HRCT between November 2016 and November 2017 were prospectively enrolled. Twelve were affected by polymyositis and 9 by dermatomyositis. We collected smoking habits data and myositis specific autoantibodies positivity, respiratory symptoms according to MRC dyspnea scale and two patients reported outcome questionnaires: Leicester cough questionnaire (LCQ) and St. George's Respiratory questionnaire (SGRQ). Patients underwent a non-contrast, supine volumetric $\mathrm{CT}$ ( $\leq 2 \mathrm{~mm}$ slice thickness) that was evaluated by an expert radiologist according to Warrick's score (WS) and also quantified by CALLIPER (Mayo Biomedical Imaging Resource - USA). CALLIPER analysis of the extent of interstitial lung abnormalities (ground glass, lung reticulation, honeycombing) was performed and a total score, expressed as percentage of lung involved (ILD\%), was obtained. Analysis of the vascular involvement were performed as percent of pulmonary vessel volume (VESS\%).

Results: Between the 21 patients, five $(23.8 \%)$ were smokers. Four patients were positive for anti-synthetase autoantibodies -ASS ( 3 Jo-1, 1 Pl-7), 1 SRP, 1 Mi2. Dyspnea was present in 19 patients (90\%): 9 grade 1,7 grade 2,3 grade 3 according to MRC scale. There were strict correlations between WS and ILD $(r=0.802 p<0.001)$ and VESS\% $(r=0.746 p<0.001)$. LCQ score was negative correlated to WS ( $r=-0.728 p=0.007)$ but the correlations were stricter with ILD score $(r=-0.868 p<0.001)$ and VESS $\%(r=-0.801 p<0.002) . S G R Q$ did not correlate both to WS and CALLIPER parameters. Patients with ASS have higher ILD and VESS $\%$ compared to seronegative patients (respectively $p=0.003$ and $p=0.006$ ). Conclusions: CALLIPER represents an innovative technique to quantify the lung involvement in IIM patients: the main advantage of CALLIPER is the reproducibility that avoids the inter and intra-reader bias. Although more data are needed in larger cohorts, the use of CALLIPER may open new routes for the evaluation of ILD in IIM patients, both in medical practice and in randomised controlled trials.

Disclosure of Interest: None declared DOI: 10.1136/annrheumdis-2018-eular.4265

\section{FRI0466 \\ ARTICULAR AFFECTION IN SYSTEMIC SCLEROSIS: CORRELATION OF ULTRASOUND FINDINGS WITH CLINICAL, BIOLOGICAL AND RADIOGRAPHICAL FINDINGS}

C. De la Puente, J. Quiñones, S. Zegarra. University Hospital Ramón y Cajal., Madrid, Spain

Objectives: To evaluate the prevalence of subclinical synovitis in systemic sclerosis (SS), as well as the correlation of sonographic findings in hands with radiographs and with clinical-analytical parameters. In addition, the correlation with scales of functionality will be determined.

Methods: Cross-sectional observational-analytical study that included 40 patients with ES and 23 patients with rheumatoid arthritis (RA) as a control group during the period 10/2015-03/2016. Clinical, analytical, immunological, ultrasound and the radiological characteristics of both groups and their respective statistical correlations were compiled.

Results: The mean age of the group with SS was $53.3 \pm 14.1$ years; $87.5 \%$ were women and $75 \%$ of the cases were cutaneously limited. The average time of evolution of the disease was 6.8 years from the diagnosis. Regarding the clinical joint involvement in the carpus and hands, frank arthritis was present in 16 cases $(40 \%)$, with an average of DAN: 3.77 and TAN: 1.57 ; with an average of EVA global pain: $30 / 100$ and elevated CRP in $42.5 \%$ of cases. The prevalence of subclinical synovitis with ultrasound expression of synovial effusion \pm synovial hyperrtrophy in the SS group was at carpal level (77\%), FCM (60\%), PFI $32.5 \%)$ and DFI (15\%). The statistical linear correlation between findings of cortical erosions at the ultrasound level versus the radiological data was positive only in the involvement at the joint level FCM and FID. In the group with SS, the HAQ questionnaire 